Meta

Journal des traducteurs

Translators' Journal

\title{
Le rôle des internationalismes dans la formation des traducteurs
}

\section{Reiner Arntz}

Volume 33, numéro 4, décembre 1988

Symposium AILA 1987, Sydney

URI : https://id.erudit.org/iderudit/001906ar

DOI : https://doi.org/10.7202/001906ar

Aller au sommaire du numéro

Éditeur(s)

Les Presses de l'Université de Montréal

ISSN

0026-0452 (imprimé)

1492-1421 (numérique)

Découvrir la revue

Citer cet article

Arntz, R. (1988). Le rôle des internationalismes dans la formation des traducteurs. Meta, 33(4), 497-506. https://doi.org/10.7202/001906ar
Résumé de l'article

Les internationalismes de langues de spécialité, en particulier ceux d'origine gréco-latine, peuvent certes être une aide pour le traducteur technique; mais ce dernier se doit toutefois d'éviter les erreurs que peut entraîner l'utilisation irréfléchie de termes étrangers, ce que quelques exemples mettent en lumière. La suite de l'article, s'appuyant sur un modèle didactique concret, explique comment intégrer, dans le cadre d'un cursus pour traducteurs techniques, une introduction systématique aux formants internationaux. L'étude systématique des formants internationaux aide l'étudiant à comprendre des termes jusque-là inconnus tout en représentant un support important à la mémorisation des terminologies. En outre, la comparaison critique entre termes de différentes langues lui montre que l'identité de la forme ne permet pas de conclure sans réserve à l'identité du contenu.
Ce document est protégé par la loi sur le droit d'auteur. L'utilisation des services d'Érudit (y compris la reproduction) est assujettie à sa politique d'utilisation que vous pouvez consulter en ligne.

https://apropos.erudit.org/fr/usagers/politique-dutilisation/ 


\section{LE RÔLE DES INTERNATIONALISMES DANS LA FORMATION DES TRADUCTEURS}

REINER ARNTZ

Hochschule Hildesheim, Allemagne fédérale

\section{RÉSUMÉ}

Les internationalismes de langues de spécialité, en particulier ceux d'origine gréco-latine, peuvent certes être une aide pour le traducteur technique ; mais ce dernier se doit toutefois d'éviter les erreurs que peut entraîner l'utilisation irréfléchie de termes étrangers, ce que quelques exemples mettent en lumière.

La suite de l'article, s'appuyant sur un modèle didactique concret, explique comment intégrer, dans le cadre d'un cursus pour traducteurs techniques, une introduction systématique aux formants internationaux.

L'étude systématique des formants internationaux aide l'étudiant à comprendre des termes jusque-là inconnus tout en représentant un support important à la mémorisation des terminologies. En outre, la comparaison critique entre termes de différentes langues lui montre que l'identité de la forme ne permet pas de conclure sans réserve à l'identité du contenu.

\section{INTRODUCTION}

Ces dernières décennies, l'importance des vocabulaires scientifiques et techniques a considérablement augmenté, et elle continue d'ailleurs à grandir. C'est, en partie du moins, à cause de l'étroitesse toujours accrue des relations internationales qu'une grande partie des termes nouvellement formés sont ce qu'on appelle des internationalismes.

Si l'on en croit Wüster, un internationalisme est une dénomination qui existe dans plusieurs langues sous la même forme ou sous une forme analogue. Ce sont essentiellement des néologismes dont les formants sont surtout d'origine grecque ou latine. Dans l'idéal, l'existence de ces néologismes et leur usage international remonte à une convention passée entre spécialistes de différents pays; mais dans la réalité il arrive beaucoup plus souvent que de tels néologismes soient apparus d'abord dans une langue naturelle particulière, par exemple l'anglais, et qu'ensuite ils soient passés dans d'autres langues.

\section{LES INTERNATIONALISMES DANS UNE PERSPECTIVE LINGUISTIQUE ET TRADUCTOLOGIQUE}

Quelle est l'attitude de la linguistique vis-à-vis de ces internationalismes de spécialité ? Il faut bien reconnaître que pendant très longtemps elle ne s'est guère posée ce problème, pas plus d'ailleurs que celui des terminologies en général. On partait du principe qu'il ne s'agissait là que de nomenclatures qui se contentaient d'étiqueter de façon unifiée sur le plan international les choses et les notions.

Pour ce qui est de la langue générale, il y a relativement peu de travaux sur ces internationalismes, et ce malgré leur importance ; et ce qui a été fait porte essentiellement 
sur les faux amis, dans une perspective contrastive. Ce n'est que récemment que s'est développée une interlinguistique ayant pour objet la langue générale et l'influence réciproque entre les langues, principalement au niveau du lexique et de la syntaxe.

Or, les recherches menées par cette science encore jeune qu'est la terminologie ont mis en évidence certaines divergences structurales et sémantiques, d'une langue à l'autre, dans ces vocabulaires internationaux de spécialité que l'on croyait si homogènes, mêrne quand les signifiants ont une forme internationale. Eugen Wüster parle à ce propos de pseudo-internationalismes. De telles divergences sémantiques peuvent gêner la compréhension, justement en raison de l'identité formelle des signifiants. Par ailleurs, il arrive que les internationalismes ne passent que dans certaines langues et que, dans une langue déterminée, le mot international d'origine gréco-latine se trouve en concurrence avec un mot indigène. En d'autres termes, les difficultés que pose le phénomène des faux amis à la communication générale apparaissent aussi sous une autre forme dans la communication technique internationale. Cependant, les difficultés de communication dans les langues de spécialité sont d'autant plus fâcheuses qu'elles ont des implications économiques. C'est pourquoi, pour assurer une communication internationale efficace, les normes terminologiques de l'ISO plaident résolument pour le développement systématique de vocabulaires de spécialité unifiés sur le plan international à partir de systèmes notionnels eux-mêmes unifiés.

Celui qui cherche des inventaires systématiques des morphèmes grecs ou latins servant à la constitution de terminologies en trouvera de deux sortes. On a d'une part des recueils plus ou moins exhaustifs de morphèmes et de règles de composition terminologique qui trouvent leur application dans de très nombreux domaines. Deux exemples belges : la liste de morphèmes publiée par $\mathrm{G}$. Lurquin dans le Langage et l'homme et celle qu'ont publiée H. Leclercq et S. Byl sous le titre Synoptische Lijst - Nederlandse wetenschappelijke termen van Griekse wortels afgeleid (Louvain, 1971). D'autre part, il existe des descriptions systématiques de morphèmes et de règles de composition valables pour des domaines spécifiques, particulièrement en médecine et dans les sciences de la nature. La recherche terminologique apporte à ces travaux de nature essentiellement descriptive un complément irremplaçable en posant le problème de l'internationalisation de terminologies dans un cadre proprement théorique et en apportant les éléments d'une méthodologie de la planification linguistique.

\section{POSSIBILITÉS D'INTÉGRATION DANS LA FORMATION DES TRADUCTEURS}

Il est nécessaire que le traducteur technique confronté tout spécialement aux problèmes des terminologies internationales connaisse les morphèmes les plus importants, leur signification et leurs règles d'emploi, ce qui implique qu'il ait certaines connaissances de base en linguistique et en terminologie. Le risque d'interférences entre la forme et le contenu sera en effet moins grand si le traducteur a aussi une formation théorique. Aussi a-t-il paru souhaitable que dès sa formation initiale le traducteur soit sensibilisé à ces questions.

Cette initiation aux internationalismes ne peut évidemment pas se faire de façon isolée, mais doit être intégrée dans les parties du cursus qui s'y prêtent particulièrement. Le modèle que je vais présenter s'appuie sur les expériences que j'ai pu faire à l'Université de Hildesheim, Institut de linguistique appliquée. Cet institut forme des traducteurs techniques dans deux langues étrangères et dans deux matières scientifiques et techniques. Bien évidemment, la formation se concentre sur les exercices de traduction générale et technique d'une part, et sur les matières de spécialité d'autre part. Quant à la troisième discipline importante, il s'agit de la linguistique appliquée grâce à laquelle 
l'étudiant doit acquérir les bases théoriques et méthodiques nécessaires à son activité de traducteur. Ce cours permet ensuite aux étudiants d'assister à un séminaire de linguistique appliquée et les prépare à la rédaction du mémoire de diplôme. Le cours porte sur quatre semestres, chacun d'eux constituant le cadre d'un sujet particulier. La première partie du cours comporte une introduction à la traductologie, la deuxième partie donne un aperçu des questions de typologie des textes généraux et de spécialité. La troisième partie, quant à elle, est consacrée à la terminologie alors que la quatrième partie traite des méthodes de documentation et des aides mécaniques à la traduction.

Dès le départ, ces cours ont fait une large place aux internationalismes des langues de spécialité. Cependant, le sujet a connu progressivement une importance croissante.

Dans l'introduction à la typologie des textes, nous analysons des textes tirés de différents domaines du point de vue du fond et de la forme et nous les comparons selon divers critères. Étendues ensuite à plusieurs langues, ces comparaisons intralinguistiques et interlinguistiques apportent de multiples connaissances, en particulier sur les structures textuelles, la syntaxe et le lexique. Il apparaît ainsi que les structures lexicales de certains langages techniques dans une même langue naturelle peuvent être très différentes les unes des autres. C'est le cas par exemple de l'allemand qui utilise plus ou moins, ou même souvent les formants gréco-latins. Cela est très net si on compare un texte allemand de construction mécanique à un texte allemand de chimie. L'allemand de la construction mécanique s'est développé sur plusieurs siècles, à partir de la langue des métiers, et n'utilise encore que peu d'éléments grecs, latins ou issus d'autres langues. Par contre, la langue de la chimie qui a été conçue systématiquement au niveau international possède de nombreux formants gréco-latins. D'autres langues de spécialité, par exemple celle de l'électrotechnique, occupent quant à leur structure lexicale une position intermédiaire entre ces deux extrêmes. Des langues de spécialité plus récentes, ainsi par exemple celle de l'informatique, présentent un fort pourcentage d'internationalismes d'origine anglo-saxonne. L'étude d'une telle diversité des textes donne à l'étudiant une idée du rôle que jouent les vocabulaires internationaux dans chacune de ces langues de spécialité.

Dans le cadre de la troisième partie du cours, qui porte sur la terminologie, nous analysons soigneusement les vocabulaires en question. Nous traitons d'abord systématiquement, à l'aide d'exemples, les procédés de formation des mots techniques dont disposent en particulier l'allemand, l'anglais et le français. Nous examinons en détail, pour les trois langues, suffixes et préfixes intéressant les langues de spécialité, étant donné que leur connaissance aide considérablement à comprendre et à apprendre les terminologies. Enfin, nous comparons les possibilités des trois langues quant à la formation des mots techniques.

Cette comparaison se fait sur la base de textes parallèles qui présentent les structures lexicales des différentes langues dans un contexte identique, ce qui fait particulièrement ressortir divergences et convergences. Cela ne veut pas dire que les textes parallèles, c'est-à-dire l'original et sa traduction dans plusieurs langues, soient la recette infaillible pour l'enseignement de la traduction. Il ne fait aucun doute que, dans la plupart des cas, par exemple pour la constitution de terminologies, on ne peut se fier qu’à des textes originaux. Cependant, dans des cas précis, de bonnes traductions, faites par des locuteurs compétents dans leur langue maternelle, peuvent s'avérer très précieuses. Et ceci s'applique particulièrement aux comparaisons interlinguistiques à des fins didactiques.

On trouvera en annexe des extraits de deux de ces textes modèles. Dans les deux cas les mots et expressions qui répondent au but poursuivi ont été soulignés. Le premier texte (exemple 1) parle de questions de protection de l'environnement. C'est un do- 
maine relativement nouveau qui se développe rapidement et qui est lié à toute une série de disciplines plus anciennes, par exemple la physique, la chimie, la médecine, le droit, etc. Ce qui fait que la terminologie est hétérogène et n'est pour ainsi dire pas définitivement établie. Aussi, des textes issus de ce domaine de spécialité peuvent ouvrir des vues intéressantes sur la question des néologismes ou de la créativité lexicale. Un examen approfondi comparé des textes en question montre que sous ce rapport, il existe d'une langue à l'autre des différences considérables. Toutefois, si l'on cherche des internationalismes, au sens que j'ai indiqué, c'est-à-dire des termes à base gréco-latine ayant dans les trois langues une forme et un contenu identiques, on trouvera uniquement le terme de symptômes cardiovasculaires - kardiovaskuläre Symptome - cardiovascular symptoms (ligne 10-11).

Par contre, sur ce plan-là, le deuxième exemple, un texte de chimie, présente un tout autre aspect. Le titre, déjà, fait apparaître dans les trois versions une forte concordance lexicale en raison de l'utilisation d'internationalismes. Cependant, une étude plus approfondie du texte montrerait aussi que les structures lexicales présentent moindre degré, certes - certaines divergences. Pour ne prendre qu'un exemple, le terme allemand "Deionat " (ligne 11) correspond en anglais à "deionized water " et en français à "eau désionisée ".

\section{AIDES MÉTHODIQUES À L'APPRENTISSAGE SYSTÉMATIQUE DE FORMANTS INTERNATIONAUX}

Malgré leur aridité, ces considérations systématiques sur la formation des mots dans une ou plusieurs langues comparées sont, de l'avis des futurs traducteurs, d'un intérêt et d'une utilité certains. En effet, ceci leur montre en particulier que la connaissance des lois de développement des terminologies peut les aider considérablement à comprendre et à retenir des termes techniques jusqu'ici inconnus dans leur langue maternelle et dans la langue étrangère.

Dans ce contexte, on voit toute l'importance des morphèmes internationaux grecs et latins. C'est pourquoi, d'ailleurs, beaucoup d'étudiants s'estiment désavantagés de ne pas avoir acquis, pendant leur scolarité, de connaissances fondamentales en langues mortes. Alors que, pour ce qui est du latin, cette lacune peut être comblée par une étude intensive des langues romanes, et dans une certaine mesure, de l'anglais, les étudiants, en règle générale, se trouvent désarmés devant les morphèmes grecs qui sont, souvent, bien plus importants. C'est la raison pour laquelle nous avons eu l'idée d'un cours de travaux pratiques qui initierait systématiquement les étudiants aux caractéristiques structurelles de la formation des mots grecs et latins et aux morphèmes les plus importants. Ce cours d'un semestre, facultatif, a déjà eu lieu une fois au stade expérimental.

Dans mes préparations, je me suis servi de deux ouvrages conçus en Suède dans le cadre d'une matière appelée "notions linguistiques de base " : un ouvrage de E. Wikén

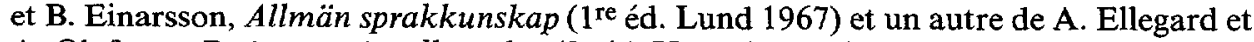
A. Olofsson, De internationella orden ( $3^{e}$ éd. Uppsala 1982). La matière en question est au programme depuis les années soixante et a pour but d'inculquer aux lycéens les bases des langues mortes qui leur permettront de comprendre et d'apprendre les éléments internationaux de leur langue maternelle et des langues vivantes modernes. Bien que le public concerné ne soit pas le même, on constate une grande similitude dans les objectifs. Ces ouvrages suédois font une large place à des listes alphabétiques des principaux formants latins et grecs. Ceux-ci sont d'abord expliqués quant à leur origine et à leur signification, puis concrétisés à l'aide d'internationalismes sous la forme qu'ils revêtent en suédois, en anglais, en français et en allemand. Ce sont ces listes que nous avons utilisées avec nombre d'adaptations et de compléments et en remplaçant les exemples tirés de la 
langue générale par des termes techniques. L'exemple donné en annexe (exemple 3) et pris dans la liste des morphèmes grecs montre que malgré tout les listes se cantonnent aux principaux éléments de formation de mots, soit 150 environ pour le grec. Ce choix limité est voulu, afin que les étudiants puissent effectivement se familiariser avec les morphèmes en question et les retenir tous. D'autre part, dans la bibliographie, nous signalons aux étudiants des listes et des tableaux plus complets qui peuvent certes apporter des informations bien plus précises, mais inadaptées à l'enseignement. Finalement, nous avons pu constater qu'une introduction systématique à un nombre limité de morphèmes internationaux particulièrement importante prépare le mieux à une consultation fructueuse d'ouvrages de référence plus complets.

Il est évident que l'analyse systématique de listes de mots, de quelque nature qu'elles soient, pose des problèmes didactiques. Afin d'éviter la monotonie et l'ennui et en même temps d'améliorer la mémorisation, il est bon de pratiquer la lecture extensive de textes de spécialité pertinents et qui ont un taux particulièrement élevé d'internationalismes.

L'annexe contient des extraits de deux textes de ce type. Il s'agit dans le premier cas (exemple 4) d'un texte d'électrotechnique dont les étudiants connaissaient déjà le contenu. J'y ai souligné les internationalismes au sens large, c'est-à-dire ceux qui ne sont pas uniquement d'origine gréco-latine et $\mathrm{j}$ 'ai indiqué en marge les équivalents allemands. Ici aussi, la comparaison montre qu'en dépit des grandes convergences, il existe aussi des divergences. Ainsi par exemple, il y a bien en allemand un verbe "dopen ", qui correspond en français à "doper » (ligne 19) mais le verbe "dotieren" est plus fréquent, et celui-ci n'a pas d'équivalent en français.

De même, les autres termes ou abréviations internationales d'origine anglosaxonne vaudraient d'être analysés en détail.

Le deuxième extrait (exemple 5), qui est tiré d'un dictionnaire de la linguistique, donne toute une série de notions fondamentales, par exemple phonologie, morphologie, dialectologie, etc., qui sont utilisées sous une forme pratiquement identique et avec la même signification dans de nombreuses langues. Ici, la difficulté pour l'étudiant n'est pas tellement de trouver le bon équivalent dans sa langue maternelle ou dans une autre langue, mais bien plus, comme l'expérience l'a montré, de saisir le contenu de ces notions, c'est-à-dire de les replacer dans un contexte spécialisé. Aussi, la connaissance de la signification des éléments de base est une aide appréciable pour l'analyse des contenus d'un domaine particulier. Ceci vaut très certainement pour la linguistique qui a une grande quantité de dénominations clairement motivées. Que ces termes si "parlants" perdent beaucoup de leur transparence en raison des interprétations des différentes écoles, c'est un problème sémantique que je ne puis traiter ici.

Évidemment, des travaux pratiques de ce genre, à cause du temps limité, ne peuvent donner que les bases qui doivent être ensuite approfondies dans les différents exercices de traduction tout au long de la formation. Étant donné que les étudiants sont plus à même d'analyser des termes jusqu'ici inconnus et de mieux reconnaître les différences notionnelles entre les langues, ils tirent de ces exercices un bénéfice accru.

\section{INTERNATIONALISMES ET NORMALISATION TERMINOLOGIQUE}

Les préoccupations didactiques que j'ai décrites partent de principes descriptifs. Cela se conçoit dans la mesure où le traducteur travaille en premier lieu sur les structures langagières effectives et n'est lui-même que rarement créateur. Toutefois, l'étude des éléments internationaux de formation de mots présente aussi un aspect prescriptif. Nous touchons ici un point qui est traité en détail dans le cadre du cours de linguistique appliquée, à savoir la normalisation terminologique. Toute normalisation terminologi- 
que présuppose une étude systématique des éléments de formation de mots existants et de leurs possibilités d'emploi. Comme je l'ai dit au début, les organisations de normalisation, internationales et en partie nationales plaident ouvertement pour l'élaboration de terminologies internationales unifiées sur la base d'éléments latins et grees. Les possibilités et les limites d'une telle normalisation au niveau national et international, qui n'est d'ailleurs pas sans soulever des critiques, posent nécessairement, entre autres, des problèmes sociolinguistiques. Ainsi, l'étudiant touche du doigt des questions que doit se poser en particulier le terminologue lorsqu'il s'agit de proposer une planification de certaines terminologies ou de combler des lacunes terminologiques. Les réflexions de ce type développent en même temps l'aptitude à juger les néologismes de langues de spécialité d'après des critères objectifs quant à leur pertinence sur la forme et sur le fond. Cette aptitude est particulièrement souhaitable, étant donné que le traducteur technique a souvent à choisir, et justement dans les nouvelles terminologies, entre plusieurs synonymes concurrents et plus ou moins appropriés.

Le présent article a montré que l'analyse intensive des aspects théoriques et pratiques des internationalismes des langues de spécialité dans le cadre de la formation des traducteurs peut être bénéfique à bien des égards. La démarche didactique présentée ici en est encore au stade expérimental et ne manquera pas de subir des modifications. C'est pourquoi je serais heureux que des collègues me fassent part des expériences qu'ils ont pu faire dans ce domaine.

\section{Annexes}



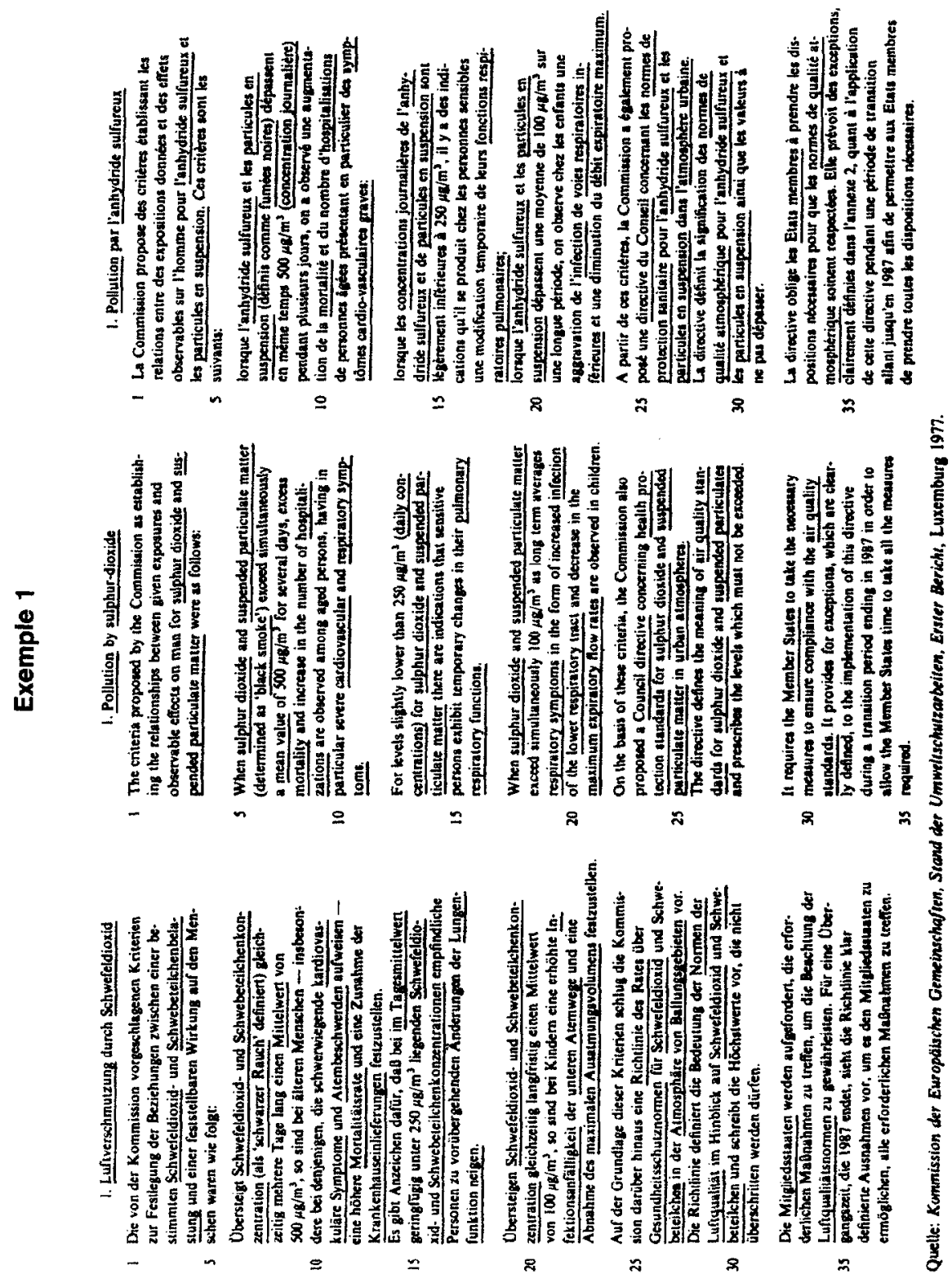

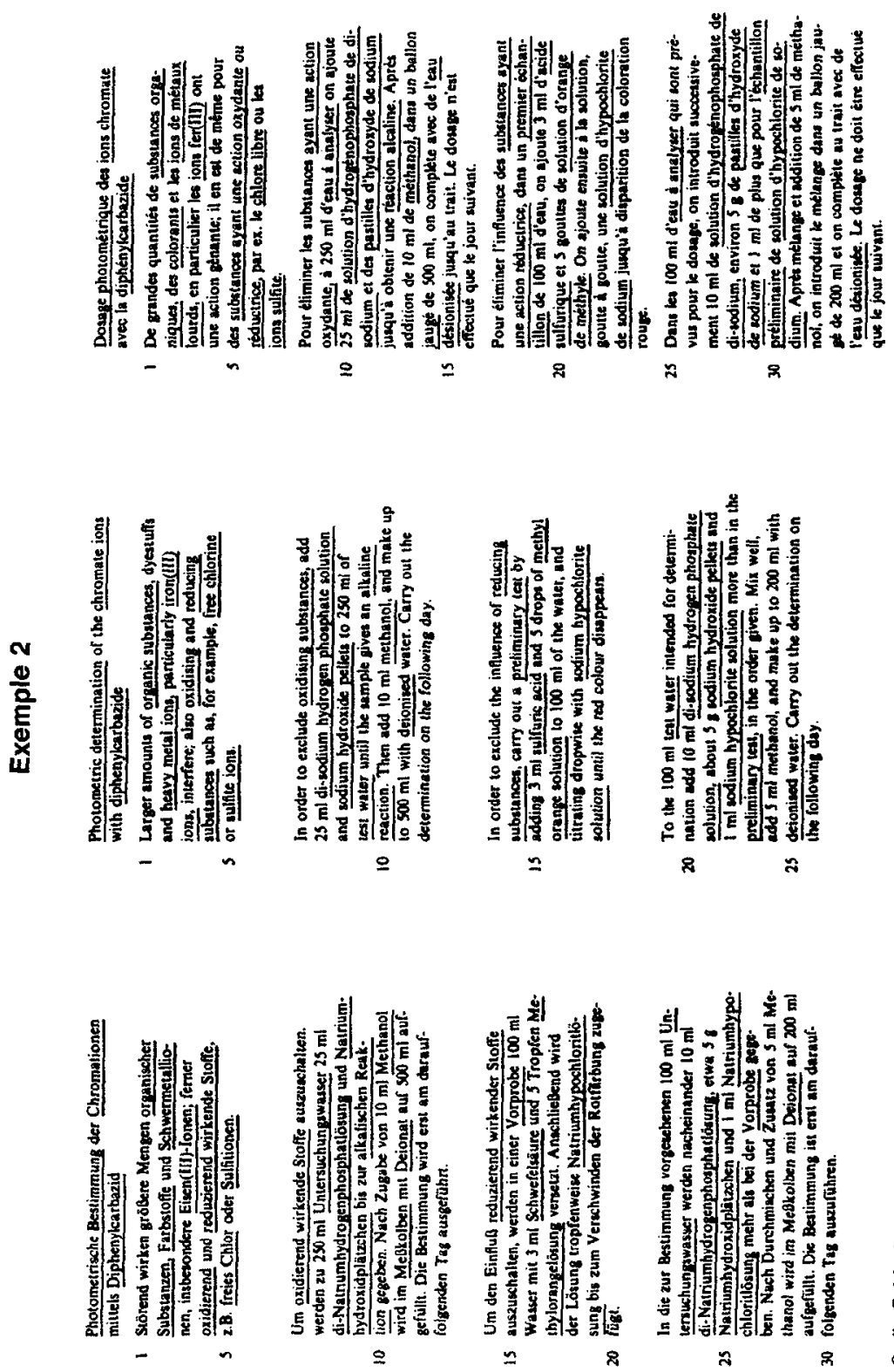

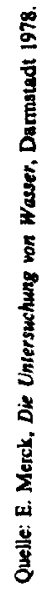




\section{Exemple 3}

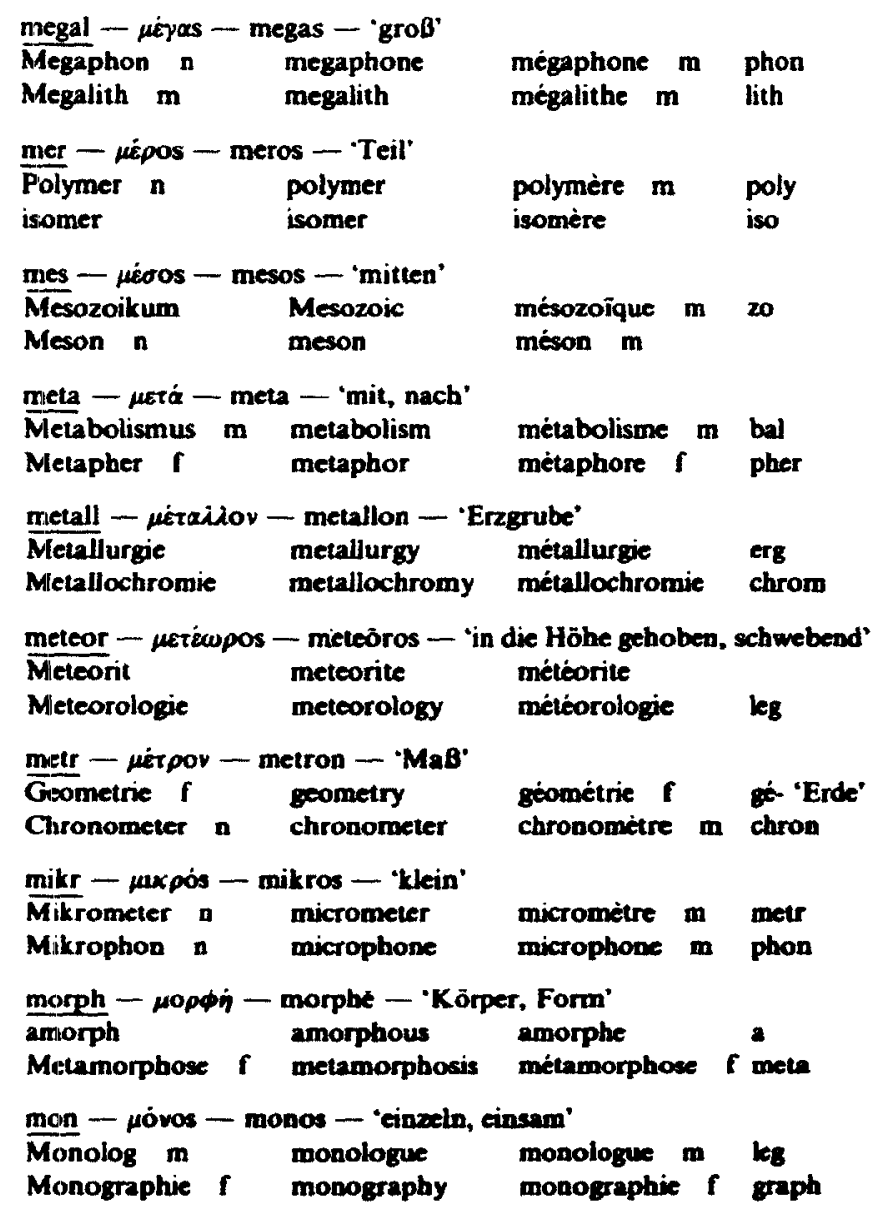




\section{Exemple 4}

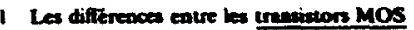
de puiscance et les bipolaira s'amenviecol. tant dans le domaine des performancet tech. niquea que des prix. Mais ave le divedoppe.

5 ment sans prixideat de la micro-thectromique.

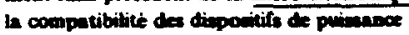
avec les microproceneruse devient une carneciristique determienante. Sas parter dea transiators bipolaines, let traniaton V-MOS

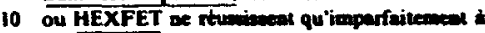
asenurer cetle compatibilite. Ce aica pas be ces des transidors SIP-MOS de Sienene Cette lochnolo pie permet en efiet it ces componans de fonctionner a un tiveau d'entrive de + 5 V/t

is $\mathrm{mA}$. Le transistor SIP-MOS $x$ rausche in famille des MOS a conduction verticale dont In structure est horizontak (uns entaille). Sur un subatrat $n^{+}$(drain), se trouve une

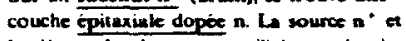

20 les élecirodes de porte en silicium polycristallin sont enterrices dans des zones $p^{*}$ et isultes par des couches d'axyde silicium. in puve forme une grilie oú les zodes de sources sont matérialisees par des trous. Pour tea

25 connexions, l'électrode de ponte est prolon. gee vers la surfuce au bord de la puce.

MOS-Leietnenarumintor Dipolartranivior

Mitrocieteronil

Mitroprodenor

Bipolartranievor MEXFET-Trasingon

SIPMOS-Trantion

a + Subatrat, Drain epitaktiech, ondotien $n^{+}$-Source, Gate-Elettrode polykristallines Silizium p*-Zone, Siliziunnoxidschicht, Chip

Quelle: Inter Electronique (Numéro spócial). Paris, April 1980.

\section{Exemple 5}

Linguistics

1 The field of study the subject of which is languge. The major branches of linguistic knowledge include phonology, grammar (morphology and syntax) and kexicology. GENERAL LINGUISTICS or THEORETICAL LINGUISTICS provides the theoretical concepts and apparatus and a common framework

5 for descriptive, historical, comparative, empirical and other types of linguistic studies, including dialectology. The term LINGUISTIC SCIENCES is used to incorporale the above as well as phonetics and sementics. The history of linguistic scholarship goes bock over many centuries, when ancient Indian and Greek linguists first obeerved

10 certain regularitics in language, e.g. between 'sounds' and 'meaning' or between 'speech' and 'writing'. Since then, linguirtics has movod away from philosophical and literary studies to become an autonomous discipline between the 'arts' and 'sciences'. Recent trends point to more intensive contacts with the eccial eciences (anthropolojical linguistics,

I5 psycholinguistics, sociolinguistica), greater use of experimental and mathematical techniques, and increasing applications to practical fields (applied linguintics, feature, philology). Alternative terma: linguistic reience, glottology (obsolete), glonsology (obeolete).

Extrait de: Hartmann, R. and F. C. Stork, Dictionary of Language and Linguistics, London: Halstedt Press, 1973. 